\title{
Sexual Functioning among Women with and without Diabetes in the Boston Area Community Health Study
}

\author{
Lauren P. Wallner, MPH, ${ }^{*}$ Aruna V. Sarma, $\mathrm{PhD}, \mathrm{MPH},{ }^{\dagger}$ and Catherine Kim, MD, $\mathrm{MPH}^{\ddagger}$ \\ *Departments of Urology and Epidemiology, University of Michigan, Ann Arbor, MI, USA; ${ }^{\dagger}$ Departments of Urology and \\ Epidemiology, University of Michigan, Ann Arbor, MI, USA; ҒDepartments of Medicine and Obstetrics and Gynecology, \\ University of Michigan, Ann Arbor, MI, USA
}

DOI: $10.1111 / \mathrm{j} .1743-6109.2009 .01510 . x$

\section{A B S T R A C T}

Aim. To examine sexual dysfunction among women with and without diabetes in a community-based sample of women aged 30-79 years.

Methods. We conducted a cross-sectional analysis of survey responses of female participants in the Boston Area Community Health Study, a community-based random sample, who answered questions regarding sexual functioning and diabetes status and also reported sexual activity $(\mathrm{n}=1,291)$. Associations between diabetes and overall sexual function as well as domain of sexual function were examined in multivariable linear regression models.

Results. Women with type 2 diabetes $(\mathrm{n}=75)$ were older, less often white, and more likely to have decreased physical activity levels, elevated body mass index, and cardiovascular disease than women without diabetes $(\mathrm{n}=1,190)$. Women with type 1 diabetes $(\mathrm{n}=26)$ were similar to women without diabetes except for higher depression scores and lower levels of activity. Age, marital status, and depressive symptoms were correlated with overall sexual function. After adjustment for age and race, women with and without diabetes had similar arousal, lubrication, orgasm, dyspareunia, satisfaction, and desire. After further adjustment for other factors, including age, depression, and marital status, women with type 1 diabetes had increased dyspareunia compared with women without diabetes, and women with type 2 diabetes had similar functioning to women without diabetes.

Conclusion. Women with type 2 diabetes may have similar sexual functioning to women without diabetes, although women with type 1 diabetes may more often have dyspareunia. Factors such as depression, which are common in women with diabetes, are more strongly related to sexual dysfunction than diabetes status. Wallner LP, Sarma AV, and Kim C. Sexual functioning among women with and without diabetes in the Boston Area Community Health Study. J Sex Med 2010;7:881-887.

Key Words. Diabetes; Women; Survey; Female Sexual Dysfunction in Diabetes

\section{Introduction}

$\mathrm{S}$ exual dysfunction is common among sexually active women, with over a third reporting dissatisfaction with overall sexual functioning, with up to $40 \%$ reporting specific sexual problems [1-3]. Compared with women without diabetes, women with diabetes face a greater proportion of disorders that may interfere with sexual function, including depression [4], vaginal candidiasis [5], and neuropathy [6].

Studies of sexual dysfunction in women are few and results conflict $[3,7-10]$. Several studies dem- onstrate a greater prevalence of sexual dysfunction among women with diabetes than women without diabetes $[9,11]$, while others do not $[3,7,12]$. Dysfunction may be more common in type 1 diabetes than in type 2 diabetes $[13,14]$, but at least one study found that dysfunction among women with type 2 diabetes was more common than in controls [8]. Also, type 2 diabetes was associated with impaired sexual functioning in case series of Nigerian women [15]. Recently, it has been hypothesized that poorer sexual functioning in women with diabetes may be related to the imbalance of sex steroid hormones [16]. 
One of the reasons for these conflicting results may be the different methods used to adjust for covariates, particularly depression and marital status. A recent report of sexual dysfunction in the Epidemiology of Diabetes Interventions and Complications study found that sexual dysfunction among women with type 1 diabetes was strongly associated with marital status and depression, but was not correlated with microvascular disease [7]. A study of Belgian women with diabetes also found that sexual dysfunction was associated with depressive symptoms but not with other factors related to diabetes [17]. These studies focused on type 1 diabetes and not on comparisons with controls.

Information on prevalence of sexual dysfunction and mediators between women with and without diabetes is limited, particularly for type 2 diabetes. Therefore, using a population-based sample, we examined the prevalence of specific aspects of sexual dysfunction in sexually active women with and without diabetes, before and after consideration of potential mediators and confounders, utilizing the Boston Area Community Health (BACH) Study.

\section{Methods}

\section{Study Population}

The study population was drawn from the BACH cohort, whose design has previously been described [1]. Briefly, the objective of BACH was to assess urologic symptoms in a community-based sample. BACH used a two-stage stratified cluster design to recruit a community-based random sample of 5,506 participants. Sampling was balanced among: males $(\mathrm{n}=2,301)$ and females $(\mathrm{n}=3,205)$, three racial/ethnic groups (black, Hispanic, and white), and four age groups (30-39, 40-49, 50-59, 60-79 years). Participants who reported sexual intercourse in the 4 weeks prior to interview were asked questions regarding sexual functioning; Of the 3,205 women in the $\mathrm{BACH}$ sample, 1,612 (50.2\%) women either did not answer the sexually active question or reported not being sexually active in the prior 4 weeks and were excluded from this analysis. Also, women who reported being both type 1 and 2 diabetic or whose diabetic status was unknown were excluded resulting in a final study population that included 1,291 sexually active women. As this study was performed on a de-identified public-use data set, it was classified as exempt by the local Institutional Review Board.

\section{Main Outcome Measures}

The BACH Female Sexual Function Index (FSFI) ascertains overall sexual function as well as function in several domains of function, including arousal, lubrication, pain, desire, orgasm, and satisfaction. All BACH participants were asked, "Over the past 4 weeks, how satisfied have you been with your overall sex life? How often did you feel sexual desire or interest? How would rate your level (degree) of sexual desire or interest?" Participants reporting intercourse in the past 4 weeks were then asked questions regarding domains of sexual function noted above. Lower scores indicate poorer sexual functioning within each domain. The 6-item overall BACH FSFI is a short-form questionnaire that was validated against the same data set used to validate the original FSFI, with a lower score reflecting worse sexual functioning [18]. Individual domain scores were examined as continuous variables and the overall BACH FSFI score was dichotomized based on the cutoff of the original FSFI, with a score of $\leq 26.2$ reflecting female sexual dysfunction (FSD) [1].

\section{Independent Variables}

Our primary exposures of interest were type 1 and type 2 diabetes. Women who reported having juvenile-onset (insulin dependent) diabetes in the $\mathrm{BACH}$ questionnaire were classified as type 1 , and women who reported having adult-onset (non-insulin dependent) diabetes were classified as type 2. Information regarding age (years), race/ ethnicity (non-Hispanic white, non-Hispanic black, Hispanic), marital status (married/living with partner, separated/divorced/widowed/, single/never married), annual household income $(<\$ 20,000, \$ 20,000-\$ 59,999, \geq \$ 60,000)$, height, weight, smoking status (never, previous, current), physical activity score $(<100,100-249, \geq 250)$, alcohol use in the past 30 days (yes/no), menopause (yes/no), cardiovascular disease (yes/no), and a modified short form of the Center for Epidemiologic Studies Depression score were obtained by interview [1]. Body mass index (BMI) was calculated and categorized as normal $(<25$ $\left.\mathrm{kg} / \mathrm{m}^{2}\right)$, overweight $\left(25-29.9 \mathrm{~kg} / \mathrm{m}^{2}\right)$, or obese $\geq 30 \mathrm{~kg} / \mathrm{m}^{2}$.

\section{Statistical Analyses}

Bivariate analyses using two-sided $t$-tests and chisquare tests for association were used to compare women with and without diabetes and women with and without FSD. Multivariable generalized linear 
models examined mean FSD domain scores by diabetes status, before and after adjustment for the variables noted above. Backward stepwise regression, which included significant covariates as determined through bivariate analyses, was used to fit a parsimonious final model. All analyses adjusted for the two-cluster complex survey design of BACH using sample weights and were performed using SAS 9.2 (Cary, NC, USA) and SUDAAN 10.0 (Research Triangle Park, NC, USA).

In sensitivity analyses, the distribution of overall and domain-specific FSD scores was examined to determine the potential for a threshold effect. No significant thresholds were observed (results not shown), so we illustrate analyses that examine outcome measures as continuous scores only.

\section{Results}

Characteristics of women with and without diabetes are illustrated in Table 1. Few women reported having type 1 diabetes $(n=26)$. Women with type 1 diabetes reported slightly lower levels of physical activity and slightly higher depression scores, indicating greater depressive symptoms when compared with women without diabetes. Women with type 2 diabetes $(\mathrm{n}=75)$ were older, less often white, and more likely to have decreased physical activity levels, elevated BMI, and cardiovascular disease than women without diabetes $(\mathrm{n}=1,190)$. Women with type 2 diabetes were also slightly more likely to report alcohol intake in the past month.

Demographic, behavioral, and comorbid characteristics by sexual dysfunction status are displayed in Table 2. Women with sexual dysfunction

Table 1 Demographic and lifestyle characteristics by diabetic status $(n=1,291)$. Unweighted frequencies (means [SE] or percentages) and weighted $P$ values shown. $P$ values compare type 1 diabetes vs. no diabetes and type 2 diabetes vs. no diabetes

\begin{tabular}{|c|c|c|c|c|c|}
\hline & $\begin{array}{l}\text { No diabetes } \\
(\mathrm{n}=1,190) \mathrm{N}(\mathrm{wt} \%)\end{array}$ & $\begin{array}{l}\text { Type } 1 \text { diabetes } \\
(n=26) N(w t \%)\end{array}$ & $\begin{array}{l}\text { Weighted } \\
P \text { value }\end{array}$ & $\begin{array}{l}\text { Type } 2 \text { diabetes } \\
(\mathrm{n}=75) \mathrm{N}(\mathrm{wt} \%)\end{array}$ & $\begin{array}{l}\text { Weighted } \\
P \text { value }\end{array}$ \\
\hline \multicolumn{6}{|l|}{ Demographic characteristics } \\
\hline Age (years) & $43.2(0.4)$ & $46.8(5.2)$ & 0.48 & $52.6(2.3)$ & $<0.01$ \\
\hline Race/ethnicity (\%) & & & 0.60 & & 0.01 \\
\hline Non-Hispanic white & $404(57.9)$ & $3(44.4)$ & & $14(28.8)$ & \\
\hline Non-Hispanic black & $401(12.0)$ & $12(18.2)$ & & $25(23.0)$ & \\
\hline Hispanic & $385(30.1)$ & $11(37.4)$ & & $36(41.2)$ & \\
\hline Marital status (\%) & & & 0.42 & & 0.24 \\
\hline Married/Living with partner & $728(65.7)$ & $16(73.6)$ & & $46(62.5)$ & \\
\hline Separated/Divorced/Widowed & $194(13.3)$ & $4(6.0)$ & & $19(23.8)$ & \\
\hline Single/Never married & $257(21.0)$ & $5(20.4)$ & & $10(13.7)$ & \\
\hline Annual household income (\%) & & & 0.14 & & 0.09 \\
\hline$<\$ 20,000$ & $489(25.2)$ & $17(41.7)$ & & $45(40.6)$ & \\
\hline$\$ 20,000-\$ 59,999$ & $414(36.5)$ & 7 (45.6) & & $21(34.1)$ & \\
\hline$\geq \$ 60,000$ & $287(38.3)$ & $2(12.7)$ & & $9(25.4)$ & \\
\hline \multicolumn{6}{|l|}{ Lifestyle characteristics } \\
\hline Body mass index (\%) & & & 0.98 & & $<0.01$ \\
\hline$<25 \mathrm{~kg} / \mathrm{m}^{2}$ & $341(40.4)$ & $2(40.2)$ & & $4(10.1)$ & \\
\hline $25-29.9 \mathrm{~kg} / \mathrm{m}^{2}$ & $365(29.6)$ & $7(31.6)$ & & $27(36.3)$ & \\
\hline$\geq 30 \mathrm{~kg} / \mathrm{m}^{2}$ & $453(30.3)$ & $15(28.2)$ & & $37(53.6)$ & \\
\hline Smoker (\%) & & & & & 0.97 \\
\hline Never & $629(52.7)$ & $18(49.5)$ & 0.97 & $38(50.6)$ & \\
\hline Previous & $275(25.4)$ & $6(23.5)$ & & $19(27.8)$ & \\
\hline Current & $286(21.9)$ & $2(27.0)$ & & $18(21.6)$ & \\
\hline Physical activity score $(\%)^{*}$ & & & 0.03 & & $<0.01$ \\
\hline$<100$ & $258(16.0)$ & $10(21.1)$ & & $39(47.6)$ & \\
\hline $100-249$ & $686(59.0)$ & $14(75.3)$ & & $31(41.5)$ & \\
\hline$\geq 250$ & $246(25.0)$ & $2(3.6)$ & & $5(10.9)$ & \\
\hline Drank alcohol in the past 30 days (\%) & & & 0.06 & & 0.04 \\
\hline No & $437(29.5)$ & $15(72.9)$ & & $40(49.0)$ & \\
\hline Yes & $753(70.5)$ & $11(27.1)$ & & $35(51.0)$ & \\
\hline \multicolumn{6}{|l|}{ Comorbidities } \\
\hline Postmenopausal (\%) & $211(58.7)$ & $6(67.2)$ & 0.67 & $35(73.3)$ & 0.18 \\
\hline Cardiovascular disease (\%) & $74(6.2)$ & $7(7.5)$ & 0.74 & $18(25.9)$ & 0.01 \\
\hline Modified 8-item CES-D score ${ }^{\dagger}$ & $1.8(0.1)$ & $4.3(0.7)$ & $<0.01$ & $2.5(0.5)$ & 0.13 \\
\hline
\end{tabular}

*Greater scores indicate greater activity.

†Greater scores indicate greater depressive symptoms.

CES-D = Center for Epidemiologic Studies Depression. 
Table 2 Demographic and lifestyle characteristics by sexual dysfunction status $(n=1,291)$

\begin{tabular}{|c|c|c|c|}
\hline & $\begin{array}{l}\text { No FSD }(>26.2) \\
(n=790) N(w t \%)\end{array}$ & $\begin{array}{l}\text { FSD }(\leq 26.2) \\
(n=501) N(w t \%)\end{array}$ & $\begin{array}{l}\text { Weighted } \\
P \text { value }\end{array}$ \\
\hline \multicolumn{4}{|l|}{ Demographic characteristics } \\
\hline Age (years) & $42.1(0.5)$ & $45.7(0.7)$ & $<0.01$ \\
\hline Race/ethnicity (\%) & & & 0.21 \\
\hline Non-Hispanic white & $240(53.3)$ & $181(61.4)$ & \\
\hline Non-Hispanic black & $265(13.1)$ & $173(11.7)$ & \\
\hline Hispanic & $285(33.7)$ & $147(26.9)$ & \\
\hline Marital status & & & 0.02 \\
\hline Married/Living with partner & 467 (61.4) & 323 (71.9) & \\
\hline Separated/Divorced/Widowed & $148(16.7)$ & $69(9.2)$ & \\
\hline Single/Never married & 169 (22.0) & $103(18.9)$ & \\
\hline Household Income & & & 0.65 \\
\hline$\leq 19,999$ & $326(26.0)$ & $225(26.2)$ & \\
\hline $20,000-59,999$ & $277(35.0)$ & 165 (38.7) & \\
\hline$>60,000$ & $187(39.0)$ & $111(35.2)$ & \\
\hline Education (year), mean (se) & $15.1(0.2)$ & $14.8(0.2)$ & 0.71 \\
\hline \multicolumn{4}{|l|}{ Lifestyle characteristics } \\
\hline Body mass index (\%) & & & 0.85 \\
\hline$<25 \mathrm{~kg} / \mathrm{m}^{2}$ & $214(37.8)$ & $133(40.4)$ & \\
\hline $25-29.9 \mathrm{~kg} / \mathrm{m}^{2}$ & $248(30.4)$ & 151 (29.3) & \\
\hline$\geq 30 \mathrm{~kg} / \mathrm{m}^{2}$ & $302(31.8)$ & $203(30.3)$ & \\
\hline Smoker & & & 0.67 \\
\hline Never & 429 (53.3) & $256(51.5)$ & \\
\hline Previous & 177 (24.2) & $123(27.5)$ & \\
\hline Current & $184(22.5)$ & $122(21.1)$ & \\
\hline Physical activity score & & & 0.30 \\
\hline$<100$ & $171(17.1)$ & $136(17.6)$ & \\
\hline $100-249$ & $442(56.4)$ & $289(61.6)$ & \\
\hline$\geq 250$ & $177(26.5)$ & 76 (20.9) & \\
\hline Drank alcohol in the past 30 days & & & 0.04 \\
\hline No & $288(27.4)$ & $204(35.6)$ & \\
\hline Yes & 502 (72.6) & $297(64.4)$ & \\
\hline \multicolumn{4}{|l|}{ Comorbidities } \\
\hline Postmenopausal (yes/no) & $121(57.8)$ & $131(62.4)$ & 0.52 \\
\hline Cardiovascular disease (yes/no) & $57(6.1)$ & $42(8.4)$ & 0.39 \\
\hline Modified 8-item CES-D score, mean (SE) & $1.5(0.1)$ & $2.3(0.2)$ & $<0.01$ \\
\hline
\end{tabular}

Note: Table includes unweighted frequencies, weighted percentages, and weighted $P$ values.

CES-D = Center for Epidemiologic Studies Depression; FSD = female sexual dysfunction.

were older $(P=0.01)$ and more likely to be married $(P=0.02)$ than women without sexual dysfunction. Also, women with sexual dysfunction had higher mean depression scores when compared to women without FSD. $(P \leq 0.01)$

Sexual function scores by domain and diabetes status, adjusted for significant demographic, behavioral, and comorbid factors, are illustrated in Table 3. Women with type 1 diabetes reported greater pain with intercourse than women without diabetes $(P=0.01)$. Women with type 2 diabetes reported better achievement of orgasm than women without diabetes $(P=0.05)$. Overall sexual functioning was not found to differ between

Table 3 Weighted Least Squares (LS) means (standard errors) for female sexual dysfunction (FSD) scores by diabetes status, adjusted for age, race, marital status, income, body mass index, menopause status, physical activity, cardiovascular disease, and depression. Greater scores indicate better function. $P$ values compare type 1 diabetes vs. no diabetes and type 2 diabetes vs. no diabetes

\begin{tabular}{|c|c|c|c|c|c|}
\hline FSD domain scores (range) & No diabetes & Type 1 diabetes & $P$ value* & Type 2 diabetes & $P$ value* \\
\hline Arousal (1.2-6) & $3.92(0.1)$ & $4.54(0.9)$ & 0.48 & $3.91(0.3)$ & 0.99 \\
\hline Lubrication (1.2-6) & $4.80(0.1)$ & $4.81(0.3)$ & 0.96 & $4.74(0.2)$ & 0.66 \\
\hline Pain $(0-6)$ & $4.80(0.1)$ & $2.91(0.8)$ & 0.01 & $5.12(0.3)$ & 0.27 \\
\hline Satisfaction (1.2-6) & $4.23(0.1)$ & $4.76(0.6)$ & 0.37 & $4.84(0.3)$ & 0.06 \\
\hline Desire $(1.2-6)$ & $3.21(0.1)$ & $3.70(0.7)$ & 0.45 & $3.05(0.2)$ & 0.55 \\
\hline Overall dysfunction (1.2-36) & $24.0(0.5)$ & $24.2(3.1)$ & 0.95 & $25.8(1.3)$ & 0.23 \\
\hline
\end{tabular}


women with type 1 or type 2 diabetes when compared with women without diabetes after adjustment for age, race, marital status, income, BMI, menopause status, physical activity, cardiovascular disease, and depression $(P=0.95,0.23)$.

\section{Discussion}

Female sexual function is an important component of quality of life, but relatively few studies have examined sexual dysfunction in women with diabetes, particularly compared with women without diabetes and in population-based samples. In this analysis of a community-based population survey, we found that women with diabetes had similar overall sexual function scores as well as functioning by domain to women without diabetes, before and after adjustment for other factors, with two exceptions. Women with type 1 diabetes reported greater dyspareunia than women without diabetes after consideration of other factors for dysfunction. Women with type 2 diabetes had marginally better orgasm scores and satisfaction than women without diabetes.

Women with diabetes could be at greater risk for sexual dysfunction due to their greater rates of depression [4,7] and neuropathy [6] compared with the general population. In addition, women with diabetes are at greater risk for vaginal infections, particularly yeast candidiasis, that may have predisposed them to dyspareunia [5]. It is possible that the sample of women with type 1 diabetes reported greater dyspareunia for this reason. Previous studies performed using $\mathrm{BACH}$ and other data have found that FSD is associated with greater age, married or partnered status, and depressive symptoms. Other factors, such as income, BMI [1], and degree of microvascular and macrovascular disease and control [7],were not significantly associated with dysfunction. While we noted greater depressive symptom scores among women with type 1 diabetes, adjustment for this and other covariates did not remove the association between type 1 diabetes and dyspareunia. Although women with type 2 diabetes more frequently had profiles that might increase their risk of sexual dysfunction, such as greater comorbid disease, sexual functioning was similar to women without diabetes before and after adjustment for these other factors.

These results contrast with nonpopulationbased studies that compared women with and without diabetes, which suggest that women with both type 1 and type 2 diabetes suffer from dys- function more frequently than women without diabetes $[2,13,14,19]$. Doruk and colleagues [13] found that Turkish women with type 1 diabetes $(n=21)$ had greater dyspareunia and lower rates of orgasm and satisfaction than women without diabetes $(n=56)$, although the difference was slight. Women with type 2 diabetes $(n=50)$ had lower rates of arousal than women without diabetes [13]. Women with diabetes were more often menopausal and reported cardiovascular disease more frequently, but comparisons did not adjust for these factors. Abu Ali and colleagues found that approximately $60 \%$ of Jordanian women with diabetes aged greater than 50 years reported sexual dysfunction, and diabetic women had lower desire, arousal, lubrication, and orgasm than women without diabetes, but comparisons adjusting for potential confounders, including marital and menopausal status, were not performed [19]. Other studies from Germany [14], Belgium $[17,20]$, and the United States [7] have found high rates of sexual dysfunction, ranging from approximately $1 / 4$ to $1 / 3$ among women with type 1 diabetes and $40 \%$ in women with type 2 diabetes, although a comparison group was not included $[7,14,17,20]$.

Our results may differ from these studies for several reasons. We examined a population-based community sample, as opposed to a clinic or hospital-based sample, and thus, the BACH participants may have been healthier despite their diagnosis of diabetes. Our results are comparable to population-based studies, suggesting that selection factors may account for significant associations between diabetes and sexual function [3]. Similarly, due to the manner in which the BACH assessed functioning across domains, we only examined women who reported sexual activity in the last 4 weeks; it is possible that if we were able to ascertain functioning in domains in women who were less sexually active, we would have detected greater differences. Also, we were able to adjust for factors associated with sexual dysfunction in general populations, particularly depressive symptoms and menopausal status, which are important predictors of dysfunction in other studies [7]. Salonia and colleagues noted that Italian women with type 1 diabetes reported similar sexual function during the follicular phase of their menstrual cycle to controls, but women with diabetes had worsened sexual function during the luteal phase [21]. Also, Veronelli and colleagues found premenopausal women with diabetes to have lower FSFI scores compared with premenopausal 
women without diabetes [12]. The BACH data were collected irrespective of menstrual cycle, and as a result, our associations may be underestimated. Similarly, at least one study in mice suggests that hormone supplementation may counteract diabetes sexual dysfunction [22], and hormone replacement (not measured in $\mathrm{BACH}$ ) may have led to unmeasured confounding, although the direction of this bias is not known. Finally, it is possible that the prevalence of sexual function in women with diabetes varies in the United States compared with other countries.

While our study is the first to recently investigate sexual functioning among women with type 2 diabetes, there are important limitations to consider. As this was a cross-sectional study, it is not possible to determine if diabetes is causally related to sexual functioning in women. Also, the number of women with type 1 diabetes was small, and we may have been underpowered to detect differences in domains of sexual functioning. The presence of diabetes and comorbidities were obtained by self-report; therefore, undetected or unreported disease may have confounded our estimates resulting in underestimated associations. The majority of women $(69 \%)$ with type 2 diabetes in this cohort reported they were not sexually active, and for that reason were not asked BACH FSFI questions. Therefore, it is possible that the amount of sexual dysfunction in women with diabetes in this sample is underestimated and is biasing our results toward the null. We did not ascertain distress associated with limited sexual functioning, and it is possible that examinations of distress might have revealed a different pattern of associations [3]. Finally, we conducted multiple comparisons, and it is possible that the slight associations between diabetes and particular domains of functioning may have occurred by chance.

\section{Conclusions}

We conclude that in a population-based sample in the United States, women with type 2 diabetes may have similar overall sexual functioning compared with women without diabetes, although women with type 1 diabetes may have greater dyspareunia than women without diabetes. These findings suggest that greater sexual dysfunction previously documented in women with diabetes may have been mediated or confounded by other factors, particularly depression or menopausal status. Further research should be conducted examining the association between depression and sexual function, particularly regarding modification by comorbid disease.

Corresponding Author: Catherine Kim, MD, MPH, Medicine, University of Michigan, 300 North Ingalls Building, Room 7C13, Mailstop 5429, Ann Arbor, MI 48109, USA. Tel: 734-936-8944; Fax: 734-647-9688; E-mail: cathkim@umich.edu

Conflict of Interest: None.

\section{Statement of Authorship}

\section{Category 1}

(a) Conception and Design

Lauren P. Wallner; Aruna V. Sarma; Catherine Kim

(b) Acquisition of Data

Catherine Kim

(c) Analysis and Interpretation of Data

Lauren P. Wallner; Aruna V. Sarma; Catherine Kim

\section{Category 2}

(a) Drafting the Article

Lauren P. Wallner; Aruna V. Sarma; Catherine Kim

(b) Revising It for Intellectual Content

Lauren P. Wallner; Aruna V. Sarma; Catherine Kim

\section{Category 3}

(a) Final Approval of the Completed Article

Lauren P. Wallner; Aruna V. Sarma; Catherine Kim

\section{References}

1 Lutfey K, Link C, Rosen R, Wiegel M, McKinlay J. Prevalence and correlates of sexual activity and function in women: Results from the Boston Area Community Health (BACH) survey. Arch Sex Behav 2008;38:514-27.

2 Ogbera AO, Chinenye S, Akinlade A, Eregie A, Awobusuyi J. Frequency and correlates of sexual dysfunction in women with diabetes mellitus. J Sex Med 2009. Jul 21 [Epub ahead of print] doi: 10.1111/j.1743-6109.2009.01396.x.

3 Shifren J, Monz B, Russo P, Segreti A, Johannes C. Sexual problems and distress in United States women: Prevalence and correlates. Obstet Gynecol 2008;112:970-8.

4 Li C, Ford E, Strine T, Mokdad A. Prevalence of depression among US adults with diabetes: Findings from the 2006 Behavioral Risk Factor Surveillance System. Diabetes Care 2008;31:105-7.

5 Bohannon N. Treatment of vulvovaginal candidiasis in patients with diabetes. Diabetes Care 1998;21:451-6.

6 Dyck P, Kratz K, Karnes J, et al. The prevalence by staged severity of various types of diabetic neuropathy, retinopathy, and nephropathy in a 
population-based cohort: The Rochester Diabetic Neuropathy Study. Neurology 1993;43:817-24.

7 Enzlin P, Rosen R, Wiegel M, et al. Sexual dysfunction in women with type 1 diabetes: Long-term findings from the DCCT/EDIC study cohort. Diabetes Care 2009;32:780-5.

8 Kolodny R. Sexual dysfunction in diabetic females. Diabetes 1971;20:557-9.

9 Schreiner-Engel P, Schiavi R, Vietorisz D, Smith H. The differential impact of diabetes type on female sexuality. J Psychosom Res 1987;31:23-33.

10 Tyrer G, Steel J, Ewing D, Bancroft J, Warner P, Clarke B. Sexual responsiveness in diabetic women. Diabetologia 1983;24:166-71.

11 Veronelli A, Mauri C, Zecchini B, et al. Sexual dysfunction is frequent in premenopausal women with diabetes, obesity, and hypothyroidism, and correlates with markers of increased cardiovascular risk. A preliminary report. J Sex Med 2009;6:1561-8.

12 Jensen S. Sexual dysfunction in younger insulintreated diabetic females. A comparative study. Diabetes Metab 1985;11:278-82.

13 Doruk H, Akbay E, Cayan S, Akbay E, Bozlu M, Acar D. Effect of diabetes mellitus on female sexual function and risk factors. Arch Andrology 2005;51:1-6.

14 Schiel R, Muller U. Prevalence of sexual disorders in a selection-free diabetic population. Diabetes Res Clin Pract 1999;44:115-21.
15 Olarinoye J, Olarinoye A. Determinants of sexual function among women with type 2 diabetes in a Nigerian population. J Sex Med 2008;5:87886.

$16 \mathrm{Kim}$ N. Sex steroid hormones in diabetes-induced sexual dysfunction: Focus on the female gender. J Sex Med 2009;6:239-46.

17 Enzlin P, Mathieu C, Van den Bruel A, Vanderschueren D, Demyttenaere K. Prevalence and predictors of sexual dysfunction in patients with type 1 diabetes. Diabetes Care 2003;26:409-14.

18 Wiegel M, Meston C, Rosen R. The Female Sexual Function Index (FSFI): Cross-validation and development of clinical cutoff scores. J Sex Marital Ther 2005;31:1-20.

19 Abu Ali R, Al Hajeri R, Khader Y, Shegem N, Ajlouni K. Sexual dysfunction in Jordanian women. Diabetes Care 2008;31:1580-1.

20 Enzlin P, Mathieu C, Van den Bruel A, Bosteels J, Vanderschueren D, Demyttenaere K. Sexual dysfunction in women with type 1 diabetes. Diabetes Care 2002;25:672-7.

21 Salonia A, Lanzi R, Scavini M, et al. Sexual function and endocrine profile in fertile women with type 1 diabetes. Diabetes Care 2006;29:312-6.

22 Cushman T, Kim N, Joyt R, Traish A. Estradiol ameliorates diabetes-induced changes in vaginal structure of db/db mouse model. J Sex Med 2009;6: 2467-79. 